\title{
我国台湾东北部龟山自附近海域热液流体中的稀士元素 组成及其对浅海热夜活动的指示
}

\author{
王晓媛 $\mathbb{1}^{*}$, 曾志刚 ${ }^{\mathbb{1}}$, 陈师 ${ }^{\mathbb{1}}$, 殷学博 ${ }^{(1)}$, 陈镇东 ${ }^{(2)}$ \\ (1) 中国科学院海洋研究所海洋地质与环境重点实验室, 青岛 266071; \\ (2) 台湾中山大学海洋地质与化学研究所, 高雄 80424 \\ * 联系人, E-mail: wangxiaoyuan@qdio.ac.cn \\ 2012-11-11 收稿, 2013-03-08 接受, 2013-06-14 网络版发表 \\ 国家自然科学基金(40906029)、国家重点基础研究发展计划(2013CB429700)、山东省自然科学杰出青年基金(JQ200913)和中国科学院海洋 \\ 研究所海洋地质与环境重点实验室开放基金(MGE2011KG11)资助
}

\begin{abstract}
摘要 热液喷口系统中的稀土地球化学被集中调查, 但是浅海热液系统中这方面的研究很少. 此次研究, 呈现了台湾东北部附近海域龟山岛热液流体中稀土元素分布的新数据. 龟山岛热液 区内黄色流体和白色流体中的总稀土( $\Sigma \mathrm{REE})$ 浓度相当, 约为 813 1212 $\mathrm{ng} / \mathrm{L}$, 与浅海海水相比 显著富集. 稀土元素球粒陨石标准化 $\left(\mathrm{REE}_{\mathrm{N}}\right)$ 分配模式显示, 龟山岛黄色流体具有轻微的负 $\mathrm{Eu}$ 异常, 白色流体没有 $\mathrm{Eu}$ 异常, 这与龟山岛热液流体的相对低温和更具氧化性组成的特点有关. 龟山岛热液流体中的轻稀土(LREE)相对重稀土(HREE)轻微富集. 黄色流体和白色流体中的稀 土元素行为与水岩相互作用时间较短有关. 此外, 黄色流体中的稀土元素分布还受到极低 $\mathrm{pH}(2.81$ 和 2.29)、流体沸腾以及自然硫沉淀形成的影响, 而白色流体则还受到细小颗粒物吸附 以及稀土与氯配合的影响.
\end{abstract}

关键词

稀土元素

热液流体

浅海

龟山岛热液区

台湾东北部
稀土元素具有相似的化学性质, 它们在地球化 学过程中作为一组元素不分离, 尽管在特殊的环境 中个别元素(例如 $\mathrm{Ce}, \mathrm{Eu}$ 等)价态的改变和 $4 \mathrm{f}$ 电子亚 层收缩(镧系收缩)引起的离子半径减小, 会导致稀土 元素在迁移过程中表现出一定的差异 ${ }^{[1 \sim 3]}$. 自然界水 系统中, 稀土元素可作为判断来源的重要依据之

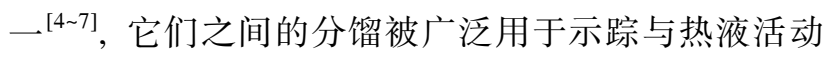
有关的许多海底过程, 例如示踪热液系统中的水-岩 相互作用 ${ }^{[8 ~ 11]}$ 、示踪热液流体的演化过程 ${ }^{[12]}$ 、示踪岩 浆酸性挥发物的脱气(例如 $\left.\mathrm{HF}, \mathrm{SO}_{2}\right)^{[7]}$ 、以及反映矿物 沉淀、溶解、离子交换和吸附 ${ }^{[13 \sim 17]}$ 等过程.

深海热液流体中的稀土元素浓度相对于周围海 水富集 1 3 个数量级 ${ }^{[18]}$, 除 Pacmanus 热液区中 satanic Mills 流体和 Fenway 流体、Desmos 热液区和
North $\mathrm{Su}$ 热液区内的酸性-硫酸盐流体、Suzette 热液 区和 South $\mathrm{Su}$ 热液区内部分黑烟图流体以及在 MAR $15^{\circ} \mathrm{N}$, MAR $5^{\circ} \mathrm{S}$ 和 MAR $9^{\circ} \mathrm{S}$ 上的一些低温扩散热液 流体之外, 其他深海热液流体均表现出一致的 $\mathrm{REE}_{\mathrm{N}}$ 配分模式, 即 LREE 富集和显著的正 Eu 异常 ${ }^{[7,14,19 ~ 23]}$. 地热流体中的稀土元素含量范围至少跨越 4 个 数量级, 从低于检测限到 $10^{-1}$ 倍球粒陨石值 ${ }^{[24]}$. 地热 流体主要有 3 种类型: (1) 低 $\mathrm{pH}$ 酸性硫酸盐流体, 这 种流体有的通过 $\mathrm{H}_{2} \mathrm{~S}$ 的大气和微生物氧化形成, 例 如新西兰 Rotokawa 酸性地热水、黄石公园中的酸性 地热流体和台湾大屯火山区中的地热流体 ${ }^{[6,25,26]}$, 有 的通过深部蒸汽 $\left(\mathrm{CO}_{2}\right.$ 和 $\left.\mathrm{H}_{2} \mathrm{~S}\right)$ 与浅部地下水混合形成, 例如新墨西哥 Valles 喷火山口中的酸性地热流体和 多米尼加酸性地热水 ${ }^{[27]}$, 还有的则是岩浆气 $\mathrm{HCl}$ 和

引用格式: 王晓媛, 曾志刚, 陈帅, 等. 我国台湾东北部龟山岛附近海域热液流体中的稀土元素组成及其对浅海热液活动的指示. 科学通报, 2013, 58: $1874-1883$

英文版见: Wang X Y, Zeng Z G, Chen S, et al. Rare earth elements in hydrothermal fluids from Kueishantao, off northeastern Taiwan: Indicators of shallow-water, sub-seafloor hydrothermal processes. Chin Sci Bull, 2013, 58, doi: 10.1007/s11434-013-5849-4 
$\mathrm{SO}_{2}$ 释放的结果, 例如新西兰 Ruapehu 弹坑湖中的 酸性热水和日本 Tamagawa 地热区中的 Obuki 酸性热 泉 $^{[11,25,28]}$; (2) 中性氯流体, 可能具有岩浆组成, 通常 代表了地热区内的深部流体，例如新西兰的 Waikite 地热流体和台湾大冈山热泉水 ${ }^{[6,29]}$; (3) 碳酸氢盐-碳 酸盐流体, 该流体通过二氧化碳水与围岩相互作用 形成, 接近中性到碱性 ${ }^{[29,30]}$, 例如台湾红叶和清水热 泉水 ${ }^{[6]}$, 以及美国俄勒冈州、内达华州和加利福尼亚 州地热区中的热液流体 ${ }^{[9]}$.

由于受到不同地质条件的影响，陆上热泉的稀 土元素分布与深海热液流体不同. 大多数酸性陆上 热泉都具有显著的“gull-wing”形状，显示负 $\mathrm{Eu}$ 异

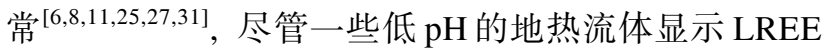
富集，且具有正 $E u$ 异常或没有 $\mathrm{Eu}$ 异常 ${ }^{[6,27]}$.

与深海热液流体和地热流体中稀土元素的研究 相比, 浅海热液流体中稀土元素的研究程度较弱, 我 们所了解的仅 Pichler 等人 ${ }^{[32]}$ 对 Tutum Bay 浅海热液 流体中的稀土元素进行过研究, 喷口流体的稀土元 素浓度比平均海水值高一个数量级, 稀土元素分布 显示出两种模式：一种为从 $\mathrm{La}$ 到 $\mathrm{Ce}$ 初始下降，随后 从 $\mathrm{Ce}$ 最小值上升到 $\mathrm{Eu}$ 最大值, 随后轻微下降到 $\mathrm{Lu}$; 另一种为从 $\mathrm{La}$ 到 $\mathrm{Ce}$ 初始下降, 然后上升到 Dy 最大 值, 随后轻微下降到 Lu.

热液流体中稀土元素的富集, 反映了热液流体 与周围岩石之间发生的水-岩相互作用 ${ }^{[7,11,15]}$, 尽管如 此, 事实和实验研究表明热液流体并未完全继承宿 主岩石中稀土元素的配分模式, 其组成主要受流体 物理化学性质(例如温度、 $\mathrm{pH}$ 、氧化还原组成、有效 配体 $)^{[19,20,26,33 \sim 36]}$ 、富含稀土元素矿物的沉淀和溶 解 ${ }^{[8,11,14,37]}$ 、二次矿物形成 ${ }^{[38]}$, 以及矿物颗粒表面吸 附 ${ }^{[16,399}$ 等因素的控制.

该研究报道了龟山岛热液流体中稀土元素的特 征，并对导致龟山岛热液区内黄色热液流体和白色 热液流体出现不同稀土元素配分模式的原因进行了 讨论. 结合前人对深海热液流体、陆上热泉以及模拟 实验研究的成果, 本次研究提供了关于热液流体中 稀土元素示踪浅海海底热液过程的较好的理解.

\section{1 地质背景}

龟山岛靠近台湾岛(距离台湾宜兰县头城镇乌石 港约 $10.8 \mathrm{~km}$ ), 位于冲绳海槽的最南端(SPOT), 归属 于琉球沟-弧系统(菲律宾海板块在欧亚大陆板块之
下俯冲形成)之后的陆内弧后盆地 ${ }^{[40]}$, 是沿冲绳海槽 扩张轴向西南延伸的最后一个火山中心 ${ }^{[41]}$, 主要由 安山岩岩浆和火成碎屑沉积物组成 ${ }^{[42]}$. 龟山岛位于 一个具有 N-S 倾向的拉张结构上, 在此处浅于 $15 \mathrm{~km}$ 的 地方存在一个清晰的地震带 ${ }^{[41]}$. 一个显著的低速带 恰好位于龟山岛下部, 暗示了此处的岩浆活动 ${ }^{[43]}$. 定年资料显示，龟山岛西北海岸火山碎屑碎岩中捕 获岩的形成年代约在 $7000 \pm 700$ 年前 ${ }^{[42]}$. 通过岛上新 鲜安山岩的微量元素、稀土元素和同位素组成 $\left({ }^{87} \mathrm{Sr} /{ }^{86} \mathrm{Sr}, \varepsilon_{\mathrm{Nd}}\right.$ 和 $\left.\delta^{18} \mathrm{O}\right)$ 的研究证实, 龟山岛处的岩浆 是弧后盆地进化过程中初始断裂阶段的产物，而且 很有可能是 MORB 型岩浆与陆壳物质同化和/或与上 覆厚层沉积物同化的结果 ${ }^{[41,44]}$.

龟山岛热液区内存在两种流体，一种为黄色流 体(温度 $92 \sim 116^{\circ} \mathrm{C}$ 之间), 另一种为白色流体(具有相 对低的温度在 $48 \sim 62^{\circ} \mathrm{C}$ 之间 $)^{[45]}$. 流体中的 $\mathrm{Mg}$ 和 $\mathrm{SiO}_{2}$ 数据显示这些流体可能来自海底之下 915 1350 m 处 ${ }^{[45]}$. 龟山岛热液流体具有高含量的 $\mathrm{CO}_{2}$ 和 $\mathrm{H}_{2} \mathrm{~S}$ 气 体以及少量的 $\mathrm{SO}_{2}$ 和 $\mathrm{HCl}$ 气体 ${ }^{[46]}$, 流体温度与潮汐 变化有关, 每一次高潮之后 2 4 小时, 流体达到它们 的最高温度 ${ }^{[47]}$. 龟山岛热液区内自然硫沉积以硫磺 砂(形似半球体, 质地软, 截面直径约为 $2 \mathrm{~mm}$, 来源 为流体喷发时落下的细粒硫磺)、自然硫烟图体( $\mathrm{S}$ 含 量很高, 达到 $99.13 \%$, 从烟图体外层向内层单质硫 含量依次增高, 该单质 $\mathrm{S}$ 来自岩浆去气作用产生的 $\mathrm{H}_{2} \mathrm{~S}$ 和 $\left.\mathrm{SO}_{2}\right)^{[48 \sim 50]}$ 和硫磺球(垒球大小, 内部剖面有年 轮状轮廓, 生长慢于自然硫烟图体, 具有 “glue pudding"生长模式)的形式存在 ${ }^{[51]}$.

\section{2 方法}

\section{1 取样方法}

龟山岛热液流体和安山岩样品于 2011 年 5 月 31 日采集，黄色流体喷口 $\left(24.8341^{\circ} \mathrm{N}, 121.9619^{\circ} \mathrm{E}\right)$ 相对 白色流体喷口 $\left(24.8349^{\circ} \mathrm{N}, 121.9619^{\circ} \mathrm{E}\right)$ 更靠近龟山岛 (图 2). 热液流体由潜水员携带容量为 $4 \mathrm{~L}$ 的 Pyrex 玻 璃瓶于龟山岛东侧海域下方采集, 每处喷口采集 2 瓶. 正常海水在龟尾水深 $10 \mathrm{~m}$ 处采集，以排除热液活动 的影响. 流体温度和流量、正常海水温度均原位测得. 流体采集方法、温度原位测定方法和流量原位测算方 法在文献[52]中详细介绍. 岩石样品由潜水员在喷口 附近使用地质锤敲击获得. 水体样品采集后, 于岸上 


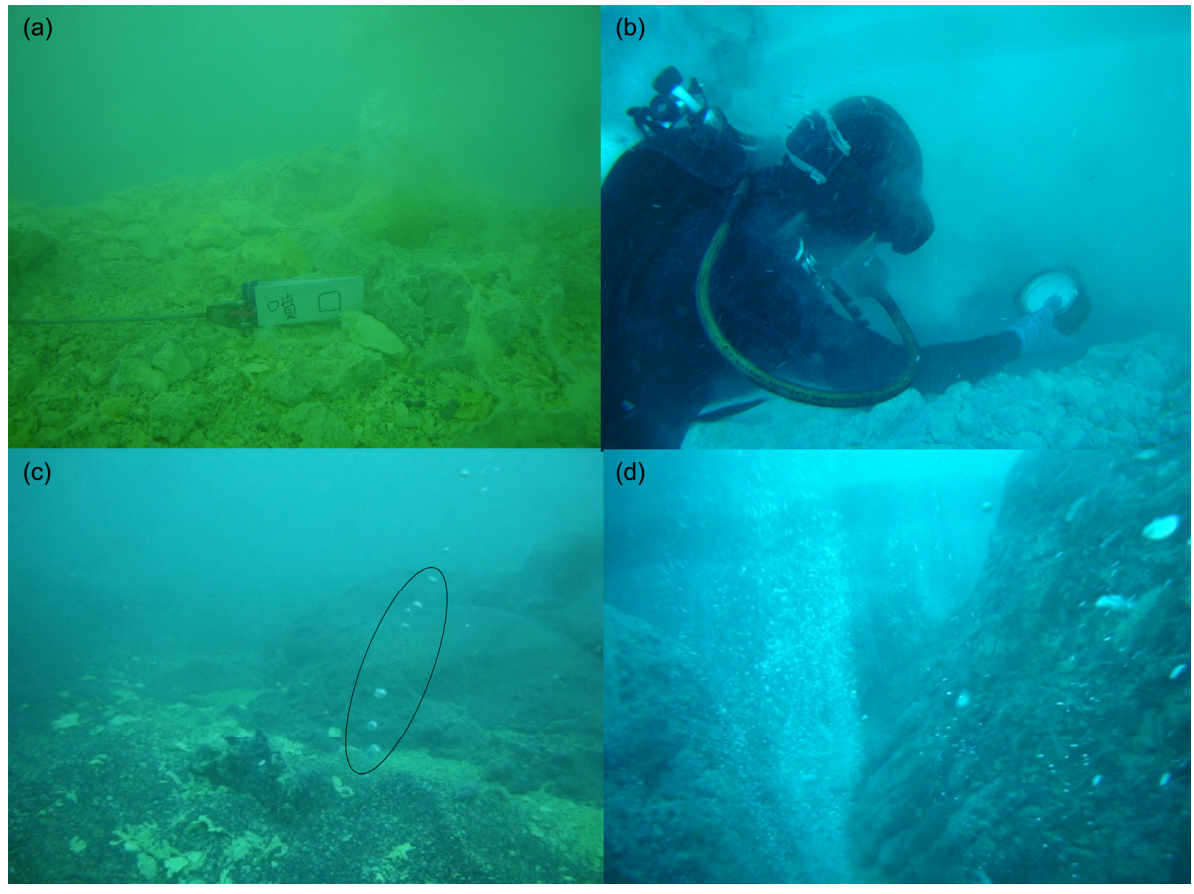

图 1 龟山岛热液区拍摄图

(a) 浊黄色流体 $\left(108^{\circ} \mathrm{C}\right.$, 流量 $35 \mathrm{~m}^{3} / \mathrm{h}$ ) 集中喷发时夹带大量硫磺颗粒, 喷口附近集结硫磺矿物; (b) 白色扩散流体喷口出露于岩石缝中, 喷出浊 白色热液 $\left(51^{\circ} \mathrm{C}\right.$, 流量 $\left.19 \mathrm{~m}^{3} / \mathrm{h}\right)$, 喷口附近没有硫磺集结, 图为潜水员正在进行原位测温; (c) 黄色流体喷口附近零星分散的气泡串(椭圆形图标圈 出); (d) 白色流体喷口附近形成的壮观的气泡墙, 反映出白色流体喷口附近的裂隙相对黄色流体喷口附近要发育

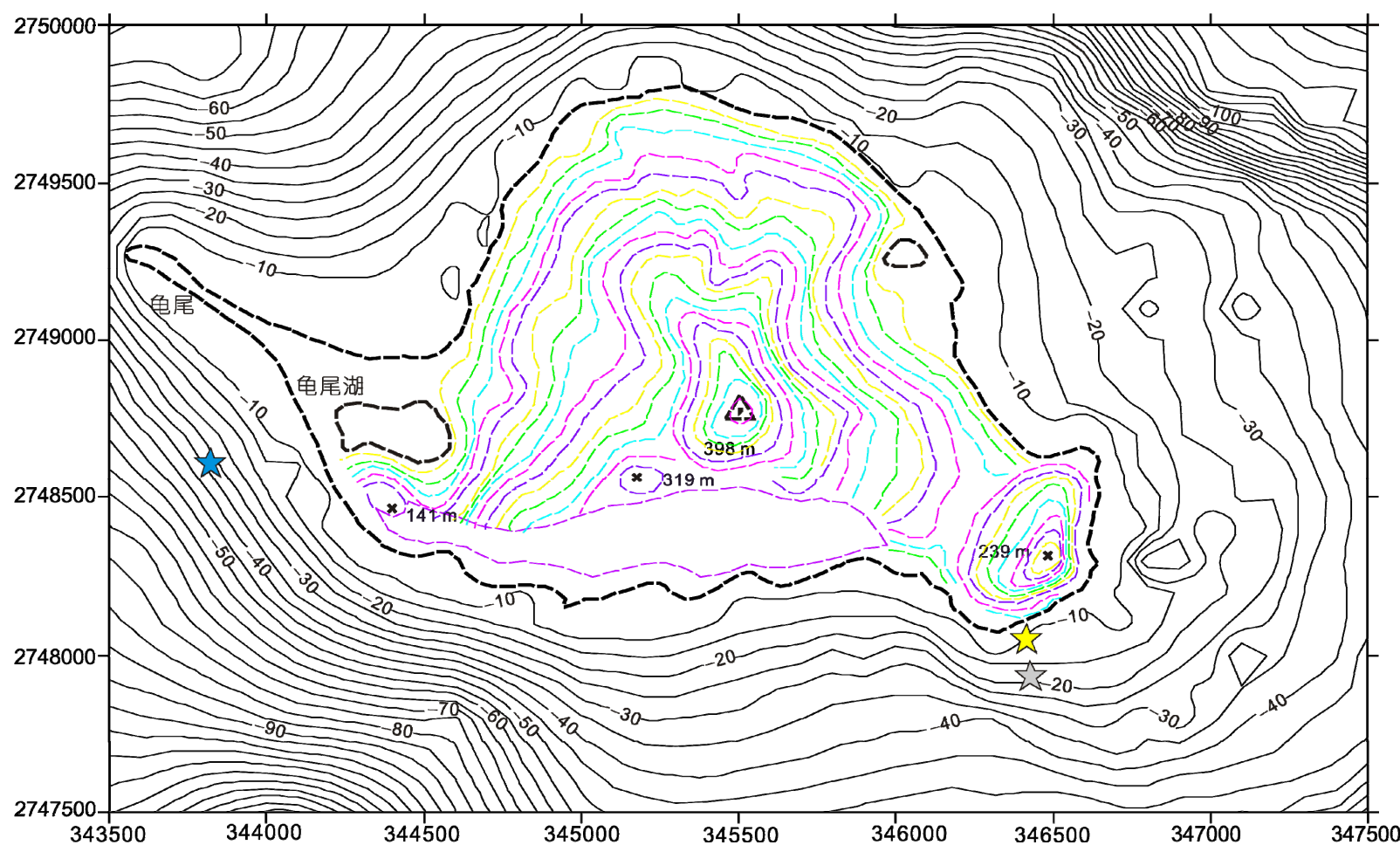

图 2 龟山岛附近海域水深图 $(\mathbf{m})$ 及采样位置

黄色流体喷口(黄色五星)位于水深 $7.2 \mathrm{~m}$ 处, 白色流体喷口(灰色五星)位于水深 $15.1 \mathrm{~m}$ 处, 两个喷口之间的直线距离为 $86 \mathrm{~m}$. 正常海水(蓝色五 星)在龟尾处采集. 此图修改自台湾探海公司提供的水深图, 横、纵坐标为 TWD 1967 TM 台湾独立坐标系统投影的平面坐标 
过滤并保存于 $1000 \mathrm{~mL}$ 的 Nalgen 塑料瓶中(样品瓶 使用前均使用 $1: 1 \mathrm{HNO}_{3}$ 浸泡 $48 \mathrm{~h}$ 之后, 依次使用蒸 馏水、纯水洗至中性并干燥).

\section{2 分析方法}

当天取完水体样品后, 立即上岸测定水样的 $\mathrm{pH}$. 水样分装至 3 个 $50 \mathrm{~mL}$ 的玻璃烧杯中, 使用美国任氏 JENCO 6010 便携式酸度计(分辨率 $0.01 \mathrm{pH}$, 温度自 动补偿)进行 $\mathrm{pH}$ 的测定, 取平均值. $\mathrm{pH}$ 计的分辨率为 0.01 , 温度自动补偿, 使用 $\mathrm{pH}$ 为 4.00 和 6.86 的缓冲 溶液标定.

水样中 $\mathrm{Cl}^{-}, \mathrm{SO}_{4}^{2-}$ 和稀土元素均在中国科学院海 洋研究所测试完成. 水样中的 $\mathrm{Cl}^{-}$和 $\mathrm{SO}_{4}^{2-}$ 使用美国戴 安公司 ICS-2500 型离子色谱仪测试, 使用乳胶型阴 离子交换树脂柱(DIONEX CS12 型)进行离子交换, 淋洗液为 $370(\mathrm{mg} / \mathrm{L}) \mathrm{Na}_{2} \mathrm{CO}_{3}-84(\mathrm{mg} / \mathrm{L}) \mathrm{NaHCO}_{3}$, 淋 洗速率为 $1.2 \mathrm{~mL} / \mathrm{min}$, 测定精密度为 $\pm 1 \%$.

水样中的 REE 使用 MetPac CC-1 树脂柱(填充材
料为亚氨基双乙酸酯, 颗粒大小约为 $17 \mu \mathrm{m}$ )富集 20 倍, 使用 $2 \mathrm{~mol} / \mathrm{L}$ 的 $\mathrm{NH}_{4} \mathrm{AC}$ 将 REE 与常量元素分离, 同时消除 $\mathrm{Ba}$ 氧化物对 $\mathrm{Eu}$ 的干扰, 最终流体样品使用 $2 \mathrm{~mol} / \mathrm{L}$ 的硝酸提取. 为提高分析的可靠性, 将纯水 (设定其中各元素浓度为 0 ) 螯合 1 倍、 $2 \mu \mathrm{g} / \mathrm{L}$ 的混标 鳌合 0.5 倍和 2.5 倍、 $20 \mu \mathrm{g} / \mathrm{L}$ 的混标鳌合 0.5 倍, 得 到 $0,1,5,10 \mu \mathrm{g} / \mathrm{L}$ 的标准溶液, 用来做 ICP-MS 的标 准曲线, 富集方法在文献[53]中进行了详细介绍. 经 过鳌合处理的水样, 使用 ICP-MS(ELAN DRCII)测试, 相对标准偏差 $<5 \%$, 测试回收率为 97\% 108\%.

水样中的 $\mathrm{Mg}$ 在中国地质调查局海洋地质实验 检测中心使用 ICP-AES 测定, 测试精密度为 $\pm 1 \%$. 像 DESMOS 和 North Su 酸性-硫酸盐流体一样, 龟山岛 热液流体在具有低 $\mathrm{pH}$ 的同时也具有高 $\mathrm{Mg}$ 浓度(表 $1)$, 外推 $\mathrm{Mg}$ 为 0 计算端员浓度是不合适的 ${ }^{[7]}$, 因此我 们未按深海热液流体外推 $\mathrm{Mg}$ 为 0 计算端员浓度的方 法来计算龟山岛热液流体的端员浓度.

在实验室中取新鲜岩石碎片, 于实体显微镜下

表 1 龟山岛黄色流体和白色流体中稀土元素的组成

\begin{tabular}{|c|c|c|c|c|c|c|}
\hline \multirow{2}{*}{ 样品 } & \multicolumn{2}{|c|}{ 黄色流体 } & \multicolumn{2}{|c|}{ 白色流体 } & \multirow{2}{*}{ 浅海海水 } & \multirow{2}{*}{ 深海海水 ${ }^{a)}$} \\
\hline & Y-1 & Y-2 & W-1 & W-2 & & \\
\hline $\mathrm{pH}\left(25^{\circ} \mathrm{C}\right)$ & 2.81 & 2.29 & 5.10 & 4.67 & 8.02 & \\
\hline $\mathrm{T}\left({ }^{\circ} \mathrm{C}\right)$ & 108 & 108 & 51 & 51 & 25 & \\
\hline $\mathrm{La}(\mathrm{ng} / \mathrm{L})$ & 128 & 185 & 183 & 179 & 8 & 4.0 \\
\hline $\mathrm{Ce}(\mathrm{ng} / \mathrm{L})$ & 287 & 413 & 422 & 407 & 11 & 0.8 \\
\hline $\operatorname{Pr}(n g / L)$ & 35 & 52 & 47 & 46 & 5 & 0.6 \\
\hline $\mathrm{Nd}(\mathrm{ng} / \mathrm{L})$ & 155 & 238 & 171 & 172 & 24 & 3.1 \\
\hline $\mathrm{Sm}(\mathrm{ng} / \mathrm{L})$ & 37 & 62 & 34 & 37 & 6 & 0.6 \\
\hline $\mathrm{Eu}(\mathrm{ng} / \mathrm{L})$ & 9 & 17 & 11 & 11 & 1 & 0.2 \\
\hline $\mathrm{Gd}(\mathrm{ng} / \mathrm{L})$ & 41 & 65 & 36 & 37 & 6 & 1.0 \\
\hline $\mathrm{Tb}(\mathrm{ng} / \mathrm{L})$ & 7 & 10 & 7 & 6 & 1 & 0.1 \\
\hline Dy(ng/L) & 42 & 63 & 49 & 47 & 10 & 1.0 \\
\hline $\mathrm{Ho}(\mathrm{ng} / \mathrm{L})$ & 9 & 13 & 11 & 10 & 2 & 0.3 \\
\hline $\operatorname{Er}(n g / L)$ & 27 & 43 & 34 & 31 & 7 & 0.9 \\
\hline $\operatorname{Tm}(n g / L)$ & 4 & 6 & 5 & 4 & 1 & - \\
\hline $\mathrm{Yb}(\mathrm{ng} / \mathrm{L})$ & 27 & 40 & 32 & 29 & 7 & 0.9 \\
\hline $\mathrm{Lu}(\mathrm{ng} / \mathrm{L})$ & 4 & 6 & 5 & 4 & 1 & 0.2 \\
\hline$\Sigma \mathrm{REE}(\mathrm{ng} / \mathrm{L})$ & 813 & 1212 & 1045 & 1022 & 92 & 14 \\
\hline$(\mathrm{La} / \mathrm{Yb})_{\mathrm{N}}$ & 2.90 & 2.84 & 3.69 & 3.88 & 0.43 & 0.23 \\
\hline$\delta \mathrm{Eu}$ & 0.74 & 0.82 & 0.95 & 0.93 & 0.59 & 0.65 \\
\hline $\mathrm{Cl}(\mathrm{mg} / \mathrm{L})$ & 17372 & 18294 & 17797 & 17301 & 19357 & 19145 \\
\hline $\mathrm{SO}_{4}(\mathrm{mg} / \mathrm{L})$ & 3007 & 3122 & 2930 & 2949 & 2709 & 2690 \\
\hline $\mathrm{Mg}(\mathrm{mg} / \mathrm{L})$ & 1050 & 1089 & 1113 & 1113 & 1147 & 1288 \\
\hline
\end{tabular}

a) 深海稀土数据引自文献[19] 
检查, 避免掺人蚀变的岩石. 用混合酸消解岩石, 在 中国科学院海洋研究所使用 ICP-MS 分析岩石中的稀 土元素组成. 测试过程中采用 BHVO-2(国际标准)、 BCR-2(国际标准)、GBW7315(国内标准)和 GBW7316 (国内标准)作为标准样品, 测试精密度除 $\mathrm{Eu}$ 为 $\pm 5 \%$ 、 $\mathrm{Lu}$ 为 $\pm 4 \%$ 之外, 其余的稀土元素在 $\pm 1 \% \sim \pm 3 \%$ 之间. 从白色流体中过滤的小颗粒, 在中国科学院生物能 源研究所使用扫描电子显微镜观测.

\section{3 结果}

\section{1 龟山岛热液流体及其中的沉淀}

由于酸性火山气的溶解和 $\mathrm{H}_{2} \mathrm{~S}$ 的氧化 ${ }^{[45]}$, 使龟 山岛黄色流体具有较低的 $\mathrm{pH}$, 此次取得黄色流体的 $\mathrm{pH}\left(25^{\circ} \mathrm{C}\right)$ 为 2.81 和 2.29 , 同时流体中的硫酸根浓度相 对浅海海水富集(表 1). 相对照, 可能由于白色泉附 近孔隙较为发育(有大量气泡溢出, 图 1(c)和图 1(d)), 海水渗人量较多, 因此白色流体的酸性被减弱, 此次 测得的白色流体的 $\mathrm{pH}\left(25^{\circ} \mathrm{C}\right)$ 分别为 5.10 和 4.67 , 白 色流体也相对浅海海水富集硫酸根(表 1).

黄色流体集中喷发后(图 1(a)), 自然硫发生沉淀, 除形成烟图体状之外, 多数在喷口附近堆积形成土 丘状, 这些黄色堆积体 (图 3(a)) 硬度小, 普通小刀刻 画即可使其破碎. 相对照, 白色流体喷口处没有形成 硫磺堆积体(图 1(b)), 但在喷口附近却弥漫着大量的 乳白色细小颗粒(图 3(b)), 通过扫描电子显微镜辨别, 这些小颗粒的主要成分为 $\mathrm{S}, \mathrm{Al}$ 和 $\mathrm{Si}$.

\section{2 龟山岛热液流体中的稀上元素特征}

龟山岛热液流体中的 $\Sigma$ REE 浓度约为 813 1212 $\mathrm{ng} / \mathrm{L}$, 比海水高 1 2 个数量级 (浅海海水 $\Sigma \mathrm{REE}$ 为 92 $\mathrm{ng} / \mathrm{L}$; 深海海水 $\Sigma \mathrm{REE}$ 为 $14 \mathrm{ng} / \mathrm{L}$ ). 黄色流体 $\mathrm{REE}_{\mathrm{N}}$ 分配模式显示 LREE 轻微富集，在 Eu 处向下有稍微 的凸起; 白色流体 $\mathrm{REE}_{\mathrm{N}}$ 分配模式也具有轻微的 LREE 富集，富集程度高于黄色热液流体，没有 $\mathrm{Eu}$ 异 常(图4). 龟山岛热液流体的 $R E_{N}$ 分配模式既不同于 浅海海水(图 4), 也不同于深海酸性-硫酸盐流体(图 5(a))、台湾大屯火山地区酸性-硫酸盐流体(图 5(b))以 及陆上酸性-硫酸盐地热流体(图 5(c)), 这可能与不同 的影响因素有关.

\section{3 龟山岛海底安山岩中的稀土元素特征}

龟山岛热液区内, 从稀土元素分布的角度分，至 少存在两种安山岩, 一种(安山岩 1) $\sum$ REE 浓度相对 较高(约为 116 137 mg/kg), 另一种安山岩(安山岩 2) 中的 $\Sigma$ REE 浓度相对较低(约为 $73 \sim 97 \mathrm{mg} / \mathrm{kg}$ ). 两种 安山岩的 $R^{2} E_{N}$ 分配模式, 均显示 LREE 富集, 但安 山岩 1 比安山岩 2 的负 $\mathrm{Eu}$ 异常更明显(图 4). 与黄色 和白色热液流体相比, 龟山岛安山岩具有较高的 LREE/HREE 比率和较大的负 Eu 异常(表 1 和 2).

\section{4 讨论}

\section{1 龟山岛热液流体中的 $\Sigma$ REE 浓度}

龟山岛热液流体中的 $\Sigma$ REE 浓度范围低于大多 数深海热液流体 ${ }^{[56]}$ 和陆上酸性热泉 ${ }^{[8,11,25,27,31]}$, 可能 是由于在龟山岛热液区海底之下流体与安山岩之间 的相互作用时间相对较短 ${ }^{[45]}$, 因而淋滤的稀土元素 含量较少.

$\mathrm{pH}$ 是控制流体中稀土元素浓度的主要因素, $\mathrm{pH}$
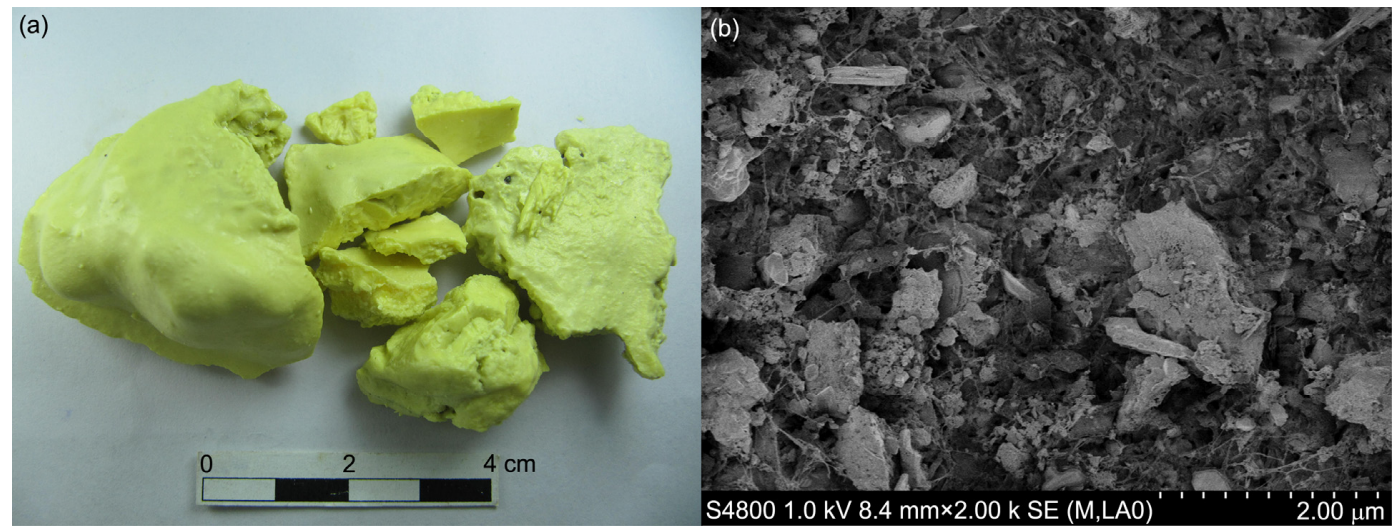

图 3 龟山岛热液区内, 黄色泉附近堆积体碎片标本图(a)与白色泉附近弥漫的细小颗粒扫描电子显微镜图(b) 


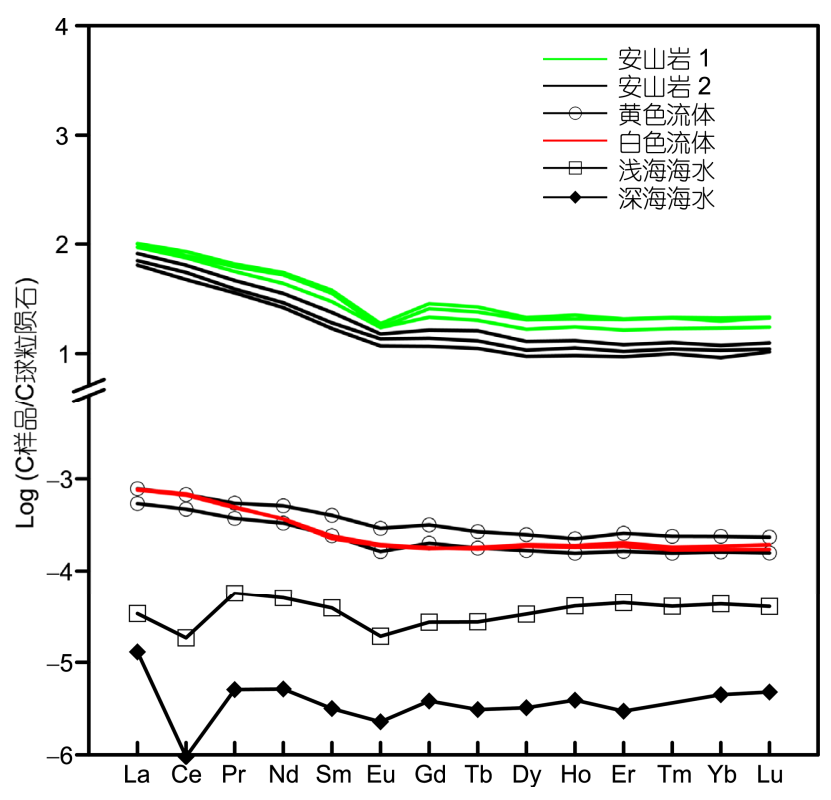

图 4 龟山岛热液流体、海底安山岩、浅海海水和深海海水 $\mathbf{R E E}_{\mathrm{N}}$ 分配模式图

大洋深海海水数据引自文献[19]. 球粒陨石数据引自文献[54]

越小, 溶液中的稀土元素浓度越高 ${ }^{[9,24,26]}$. 因此, 理 论上龟山岛黄色热液流体 $(\mathrm{pH}$ 为 2.29 和 2.81) 中的 $\Sigma \mathrm{REE}$ 浓度应该比白色热液流体 ( $\mathrm{pH}$ 为 5.10 和 4.67) 中的高. 但是，两者中的 $\Sigma$ REE 浓度相似，可能是以下 3 种过 程共同作用的结果: (1) 黄色流体集中喷发速度较快 (通量为 $35 \mathrm{~m}^{3} / \mathrm{h}$, 几乎是白色流体的 2 倍), 导致与安 山岩相互作用时间较短; (2) 流体沸腾引起溶液中的 REE 丢失 ${ }^{[9,29]}$, 龟山岛黄色流体的温度 $\left(108^{\circ} \mathrm{C}\right)$ 几乎达
到了在该深度的理论沸点 $\left(123^{\circ} \mathrm{C}\right)^{[45]} ;$ (3) 可能是黄色 泉附近形成的硫磺堆积体, 从流体中富集稀土元素, 自然硫烟图体中 $\Sigma$ REE 浓度为 $4.46 \sim 21.00 \mu \mathrm{g} / \mathrm{kg}^{[49]}$, 约是龟山岛热液流体中的 4 20 倍.

\section{2 龟山岛热液流体中的 $\delta \mathrm{Eu}$ 值}

在低温酸性条件下, 与新鲜的或轻微蚀变安山 岩相互作用的水溶液常出现负 $\mathrm{Eu}$ 异常 ${ }^{[8]}$, 例如许多 陆上酸性-硫酸盐热液流体(图 5(c)). 但是, 并不是所 有的低温酸性流体都会出现显著负 $\mathrm{Eu}$ 异常, 例如 Desmos 酸性-硫酸盐流体在 $\mathrm{Eu}$ 处有一个轻微的向上 凸起 $(\delta \mathrm{Eu}$ 为 1.18 1.31)(图 5(a))、台湾大屯火山地区 的一些酸性-硫酸盐流体在 $\mathrm{Eu}$ 处平坦 $(\delta \mathrm{Eu}$ 为 $0.94 \sim$ 1.06)(图 5(b)) 以及我们所研究的龟山岛热液流体在 $\mathrm{Eu}$ 处有轻微负异常或无异常 (黄色流体的 $\delta \mathrm{Eu}$ 为 0.74 和 0.82 , 白色流体的 $\delta \mathrm{Eu}$ 为 0.95 和 0.93)(图 4), 说明 除水岩相互作用之外, 还有其他因素影响了龟山岛 热液流体中 $\mathrm{Eu}$ 的相对浓度.

龟山岛黄色热液流体具有轻微的 $\mathrm{Eu}$ 负异常, 可 能与其相对低温 $\left(108^{\circ} \mathrm{C}\right)$ 有关. 温度是控制 $\mathrm{Eu}^{3+} / \mathrm{Eu}^{2+}$ 氧化还原电位的重要因素, 低温更利于 $\mathrm{Eu}$ 以+3 价的 形式存在 ${ }^{[34,39]}$. 而且, 稀土元素种类分布计算显示多 数酸性-硫酸盐流体 $\left(<120^{\circ} \mathrm{C}\right)$ 中 $\mathrm{REE}$ 主要以三价自由 离子的形式存在 ${ }^{[7]}$. 因此, 在低温的条件下, 龟山岛 黄色热液流体中的 $\mathrm{Eu}$ 和其他稀土元素相同, 主要表 现为 +3 价, 从而使得异常减弱或几乎消失.

白色泉附近的裂隙较为发育, 相对富氧的海水
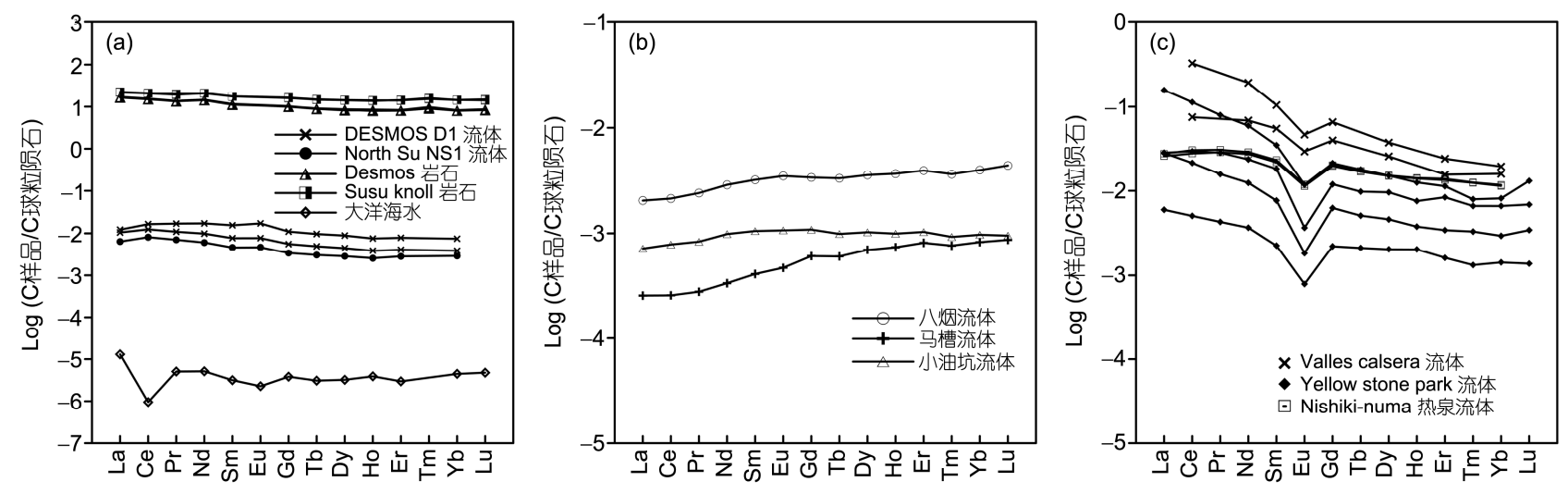

图 5 深海和地热酸性-硫酸盐流体 $\mathbf{R E E}_{\mathrm{N}}$ 分配模式图

(a) 东马努斯弧后盆地内酸性- 硫酸盐流体、围岩以及深海海水 $\mathrm{REE}_{\mathrm{N}}$ 分配模式图, 流体数据引自文献[7], 围岩数据引自文献[55], 深海海水数 据引自文献[19]; (b) 台湾大屯火山地区酸性-硫酸盐流体 $\mathrm{REE}_{\mathrm{N}}$ 分配模式图, 数据引自文献[6]; (c) 黄石国际公园、新墨西哥 Valles 喷火山口以 及日本北海道锦沼温泉酸性-硫酸盐流体的 $\mathrm{REE}_{\mathrm{N}}$ 分配模式图, 数据引自文献 $[8,27,31]$ 
表 2 龟山岛热液区海底安山岩稀土元素组成 $(\mathrm{mg} / \mathrm{kg})$

\begin{tabular}{|c|c|c|c|c|c|c|}
\hline \multirow{2}{*}{ 样品 } & \multicolumn{3}{|c|}{ 安山岩 1} & \multicolumn{3}{|c|}{ 安山岩 2} \\
\hline & K11-Y5-R1-1 & K11-Y5-R2-1 & K11-Y5-R2-3 & K11-W0-R1-1 & K11-W0-R2-1 & K11-W2-R1-1 \\
\hline $\mathrm{La}$ & 22.14 & 23.97 & 23.74 & 16.72 & 19.42 & 15.21 \\
\hline $\mathrm{Ce}$ & 45.78 & 52.25 & 48.03 & 33.69 & 39.2 & 28.98 \\
\hline $\operatorname{Pr}$ & 5.34 & 6.24 & 5.89 & 3.69 & 4.41 & 3.4 \\
\hline $\mathrm{Nd}$ & 20.39 & 25.65 & 24.44 & 13.59 & 16.51 & 12.27 \\
\hline $\mathrm{Sm}$ & 4.56 & 5.79 & 5.43 & 2.92 & 3.64 & 2.59 \\
\hline $\mathrm{Eu}$ & 1 & 1.09 & 1.01 & 0.79 & 0.88 & 0.68 \\
\hline $\mathrm{Gd}$ & 4.42 & 5.86 & 5.28 & 2.84 & 3.37 & 2.4 \\
\hline $\mathrm{Tb}$ & 0.75 & 1 & 0.9 & 0.49 & 0.6 & 0.42 \\
\hline Dy & 4.24 & 5.43 & 5.17 & 2.74 & 3.28 & 2.4 \\
\hline Ho & 0.99 & 1.27 & 1.17 & 0.64 & 0.74 & 0.54 \\
\hline $\mathrm{Er}$ & 2.72 & 3.43 & 3.38 & 1.74 & 2 & 1.55 \\
\hline $\mathrm{Tm}$ & 0.43 & 0.55 & 0.54 & 0.28 & 0.32 & 0.25 \\
\hline $\mathrm{Yb}$ & 2.91 & 3.59 & 3.36 & 1.82 & 2.02 & 1.56 \\
\hline $\mathrm{Lu}$ & 0.44 & 0.55 & 0.54 & 0.28 & 0.32 & 0.26 \\
\hline$\Sigma \mathrm{REE}$ & 116.11 & 136.65 & 128.87 & 82.22 & 96.72 & 72.52 \\
\hline$(\mathrm{La} / \mathrm{Yb})_{\mathrm{N}}$ & 5.46 & 4.79 & 5.07 & 6.57 & 6.9 & 6.99 \\
\hline$\delta \mathrm{Eu}$ & 0.68 & 0.57 & 0.58 & 0.84 & 0.77 & 0.84 \\
\hline
\end{tabular}

不断下渗, 与海底之下的流体混合, 增强了流体的相 对氧化性，降低了流体的温度，从而使龟山岛白色流 体比黄色流体更具低温 $\left(51^{\circ} \mathrm{C}\right)$ 和氧化状态的性质, 因 此, 白色流体中的 $\mathrm{Eu}$ 更是主要以 +3 价存在, 导致没 有 $\mathrm{Eu}$ 异常.

\section{3 龟山岛流体中稀上元素的配合}

龟山岛热液流体具有高 $\mathrm{Al}$ 浓度 ${ }^{[45]}, \mathrm{Al}-\mathrm{F}$ 配合限 制了稀土元素-氟配合物的形成 ${ }^{[57]}$, 而且黄色流体的 $\mathrm{pH}$ 很低, $\mathrm{F}^{-}$以 $\mathrm{HF}$ 的形式存在, 减小了作为配位用的 氟离子含量 ${ }^{[19]}$, 因此稀土元素-氟配合物对龟山岛热 液流体中的水合稀土元素种类没有贡献.

酸性硫酸盐流体中稀土元素的富集, 较少程度 上受稀土元素-硫酸根和/或稀土元素-氯配合物存在 的影响 ${ }^{[7]}$, 因此龟山岛黄色流体 (平均 $\mathrm{pH}$ 为 2.55 )中的 稀土元素分布几乎不受硫酸根和氯离子的影响. 不 过, 白色流体 $\left(51^{\circ} \mathrm{C}\right.$, 平均 $\mathrm{pH}$ 为 4.89)中的稀土元素 浓度会受到 REE-氯配合作用的影响. 模拟实验数据 显示, 在 25 和 $300^{\circ} \mathrm{C}$ 、饱和水蒸气压条件下, 轻稀土 $\left(\mathrm{La}^{3+}\right)$ 和重稀土 $\left(\mathrm{Lu}^{3+}\right)$ 与硫酸根形成的配合物的稳定 性相似 ${ }^{[58]}$, 意味着在此条件下, 稀土元素与硫酸根 的配合不会引起轻重稀土之间的分馏. 但是, 在 25 , 200 和 $300^{\circ} \mathrm{C}$, 饱和水蒸气压条件下, LREE-氯配合物
的稳定性均高于 HREE-氯配合物 ${ }^{[35,58]}$. 由此我们推 断, 稀土元素-硫酸根配合作用不会引起龟山岛白色 流体中 LREE 与 HREE 之间的分馏, 但 REE-氯配合 作用会引起白色流体中 LREE 的相对富集.

\section{4 龟山岛流体中稀上元素的分馏}

在很低的 $\mathrm{pH}($ (大约 $<1.5 \sim 2)$ 条件下, 来自岩石或 岩浆中的稀土元素之间分馏很小, 但是 $\mathrm{pH}$ 在 2 4 之 间的热水中倾向于分馏 $\mathrm{LREE}^{[25]}$. 因此, 龟山岛黄色 流体 $(\mathrm{pH}$ 为 2.29 和 2.81) 具有轻微的 LREE 富集, $(\mathrm{La} / \mathrm{Yb})_{\mathrm{N}}$ 为 3.34 和 3.28, 但是它们的 $\mathrm{REE}_{\mathrm{N}}$ 配分模式 比宿主岩石(4.79 6.99)平坦, 说明除了低 $\mathrm{pH}$, 还有 其他因素影响了黄色流体中 LREE和 HREE 之间的分 馏, 这可能与自然硫的形成有关. 自然硫中 LREE/ HREE 比值显著高于黄色流体中的值, $(\mathrm{La} / \mathrm{Yb})_{\mathrm{N}}$ 达到 6.61 9.80 $0^{[49]}$, 其 HREE 之间的分馏程度 $\left((\mathrm{Gd} / \mathrm{Yb})_{\mathrm{N}}\right.$ 为 1.10 1.48)与黄色流体(1.24 和 1.33)的相似. 可见, 受 自然硫沉淀的影响，流体中的 LREE 比 HREE 更易被 转移，从而导致黄色流体中 LREE 与 HREE 之间的分 馏减小.

龟山岛白色流体 $\left(\mathrm{pH}\right.$ 为 5.10 和 4.67)的 $\mathrm{REE}_{\mathrm{N}}$ 分 配模式也显示 LREE 相对 HREE 轻微富集, $(\mathrm{La} / \mathrm{Yb})_{\mathrm{N}}$ 为 4.13 和 4.42 , 这与喷口附近形成的乳白色细小颗 
粒密切相关. 由于 $\mathrm{pH}>4$ 的流体具有显著的颗粒稀土 元素载荷 ${ }^{[16]}$, 而且颗粒物对流体中稀土元素的吸附 (吸附强度为 $\mathrm{La}^{3+}<\mathrm{Eu}^{3+}<\mathrm{Lu}^{3+}$ ), 将使流体中 HREE 浓 度降低，导致流体稀土的分配模式具有 $(\mathrm{La} / \mathrm{Lu})_{\mathrm{N}}>1$ 的 特征 ${ }^{[39]}$.

\section{5 结论}

龟山岛热液流体中的稀土元素来源于基底安山 岩, $\Sigma$ REE 浓度低于大多数深海热液流体和陆上酸性 热泉水, 可能与海底之下水/岩相互作用时间短、流体
沸腾和流体中硫磺沉淀的形成有关. 龟山岛两种流 体的 $\mathrm{REE}_{\mathrm{N}}$ 分配模式不同于具有相似基底的深海热液 流体和陆上热泉，黄色流体 $\mathrm{REE}_{\mathrm{N}}$ 分配模式平坦，在 $\mathrm{Eu}$ 处向下有稍微的凸起; 白色流体具有轻微的 LREE 富集，没有 $\mathrm{Eu}$ 异常. 龟山岛热液流体中的 $\mathrm{Eu}$ 异常主要受控于较低的温度和相对氧化状态的性质. 龟山岛黄色流体中 LREE与 HREE之间的分馏主要受 极低 $\mathrm{pH}$ 、和自然硫沉淀形成的影响; 而白色流体中 LREE 与 HREE 之间的分馏则与颗粒物吸附和稀土元 素-Cl 配合作用有关.

\section{参考文献}

1 曾志刚, 蒋富清, 秦蕴珊, 等. 冲绳海槽中部 Jade 热液活动区中块状硫化物的稀土元素地球化学特征. 地质学报, 2001, 75: 244-249

2 Dubinin A V. Geochemistry of rare earth elements in the Ocean. Lithol Miner Resour, 2004, 39: 289-307

3 Chudaev O, Chudaeva V, Sugimori K, et al. Composition and origin of modern hydrothermal systems of the Kuril island arc. Indian J Mar Sci, 2008, 37: 166-180

4 Elderfield H. The oceanic chemistry of the rare-earth elements. Phil Trans R Soc Lond A, 1988, 325: 105-126

5 Zhang J, Nozaki Y. Behavior of rare earth elements in seawater at the ocean margin: A study along the slopes of the Sagami and Nankai troughs near Japan. Geochim Cosmochim Acta, 1998, 62: 1307-1317

6 叶怡伶. 台湾地区温泉水中稀土元素之浓度及分布. 硕士学位论文. 台南: 成功大学, 2008. 34-60

7 Craddock P R, Bach W, Seewald J S, et al. Rare earth element abundances in hydrothermal fluids from the Manus Basin, Papua New Guinea: Indicators of sub-seafloor hydrothermal processes in back-arc basins. Geochim Cosmochim Acta, 2010, 74: 675-683

8 Bau M, Usui A, Pracejus B, et al. Geochemistry of low-temperature water-rock interaction: Evidence from natural waters, andesite, and iron-oxyhydroxide precipitates at Nishiki-numa iron-spring, Hokkaido, Japan. Chem Geol, 1998, 151: 293-307

9 Wood S A, Shannon W M. Rare-earth elements in geothermal waters from Oregon, Nevada, and California. J Solid State Chem, 2003, 171: 246-253

10 刘炎光, 孟宪伟, 付云霞. 冲绳海槽中部 Jade 热液场烟图物稀土元素和锶、钕同位素地球化学特征. 海洋学报, 2005, 27: 67-72

11 Sanada T, Takamatsu N, Yoshiike Y. Geochemical interpretation of long-term variations in rare earth element concentrations in acidic hot spring waters from the Tamagawa geothermal area, Japan. Geothermics, 2006, 35: 141-155

12 陶春辉, 李怀明, 黄威, 等. 西南印度洋脊 $49^{\circ} 39^{\prime} \mathrm{E}$ 热液区硫化物烟图体的矿物学和地球化学特征及其地质意义. 科学通报, 2011, 56: $2412-2423$

13 Sverjensky D A. Europium redox equilibria in aqueous solution. Earth Planet Sci Lett, 1984, 67: 70-78

14 Klinkhammer G P, Elderfield H, Edmond J M, et al. Geochemical implications of rare earth element patterns in hydrothermal fluids from mid-ocean ridges. Geochim Cosmochim Acta, 1994, 58: 5105-5113

15 丁振举, 刘从强, 姚书振, 等. 海底热液系统高温流体的稀土元素组成及其控制因素. 地球科学进展, 2000, 15: 307-312

16 Wood S. The hydrothermal geochemistry of the rare earth elements. Gangue, 2004, 81: 1-7

17 包申旭, 周怀阳, 彭晓䚲, 等. Juan de Fuca 洋脊 Endeavour 段热液硫化物稀土元素地球化学特征. 地球化学, 2007, 36: 303-310

18 Mitra A, Elderfield H, Greaves M J. Rare earth elements in submarine hydrothermal fluids and plumes from the Mid-Atlantic Ridge. Mar Chem, 1994, 46: 217-235

19 Douville E, Bienvenu P, Charlou J L, et al. Yttrium and rare earth elements in fluids from various deep-sea hydrothermal systems. Geochim Cosmochim Acta, 1999, 63: 627-643

20 Douville E, Charlou J L, Oelkers E H, et al. The rainbow vent fluids $\left(36^{\circ} 14^{\prime} \mathrm{N}\right.$, MAR): The influence of ultramafic rocks and phase separation on trace metal content in Mid-Atlantic Ridge hydrothermal fluids. Chem Geol, 2002, 184: 37-48 
21 Schmidt K, Koschinsky A, Garbe-Schönberg D, et al. Geochemistry of hydrothermal fluids from the ultramafic-hosted Logatchev hydrothermal field, $15^{\circ} \mathrm{N}$ on the Mid-Atlantic Ridge: Temporal and spatial investigation. Chem Geol, 2007, 242: 1-21

22 Hongo Y, Obata H, Gamo T, et al. Rare earth elements in the hydrothermal system at Okinawa Trough back-arc basin. Geochem J, 2007 , 41: 1-15

23 Bau M, Balan S, Schmidt K, et al. Rare earth elements in mussel shells of the Mytilidae family as tracers for hidden and fossi high-temperature hydrothermal systems. Earth Planet Sci Lett, 2010, 299: 310-316

24 Shannon W M, Wood S A, Brown K, et al. Behavior of rare earth elements in geothermal systems: A new exploration/exploitation tool? In: Exploration Technology, Geothermal Energy R\&D Program, 2001. 2-31

25 Wood S A. Rare earth element systematics of acidic geothermal waters from the Taupo Volcanic Zone, New Zealand. J Geochem Explor, 2006, 89: 424-427

26 Lewis A J, Komninou A, Yardley B W D, et al. Rare earth element speciation in geothermal fluids from Yellowstone National Park, Wyoming, USA. Geochim Cosmochim Acta, 1998, 62: 657-663

27 Michard A. Rare earth element systematics in hydrothermal fluids. Geochim Cosmochim Acta, 1989, 53: 745-750

28 Yoshiike Y. Variation in the chemical composition of Obuki Spring, Tamagawa Hot Springs (1951-2000). Geochem J, 2003, 37: 649-662

29 Shannon W M, Wood S A, Brown K, et al. REE contents and speciation in geothermal fluids from New Zealand. In: Proceedings of the 10th International Symposium on Water-Rock Interaction. Villasimius: Balkema, 2001. 1001-1004

30 Arnórsson S, Stefánsson A, Bjarnason J. Fluid-Fluid Interactions in Geothermal Systems. Rev Mineral Geochem, 2007, 65: 259-312

31 Lewis A J, Palmer M R, Sturchio N C, et al. The rare earth element geochemistry of acid-sulphate and acid-sulphate-chloride geothermal systems from Yellowstone National Park, Wyoming, USA. Geochim Cosmochim Acta, 1997, 61: 695-706

32 Pichler T, Veizer J, Hall G E M. The chemical composition of shallow-water hydrothermal fluids in Tutum Bay, Ambitle Island, Papua New Guinea and their effect on ambient seawater. Mar Chem, 1999, 64: 229-252

33 Migdisov A A, Willianms-Jones A E, Wagner T. An experimental study of the solubility and speciation of the Rare Earth Elements(III) in fluoride- and chloride-bearing aqueous solutions at temperatures up to $300^{\circ} \mathrm{C}$. Geochim Cosmochim Acta, 2009, 73: 7087-7109

34 Hass J R, Shock E L, Sassani D C. Rare earth elements in hydrothermal systems: Estimates of standard partial molal thermodynamic properties of aqueous complexes of the rare earth elements at high pressures and temperatures. Geochim Cosmochim Acta, 1995, 59: $4329-4350$

35 Gammons C H, Wood S A, Youning L. Complexation of the rare earth elements with aqueous chloride at $200{ }^{\circ} \mathrm{C}$ and $300^{\circ} \mathrm{C}$ and saturated water vapor pressure. Geol Soc Spec Publ, 2002, 7: 191-207

36 Gammons C H, Wood S A, Williams-Jones A E. The aqueous geochemistry of the rare earth elements and yttrium: VI Stability of neodymium chloride complexes from 25 to $300^{\circ} \mathrm{C}$. Geochim Cosmochim Acta, 1996, 60: 4615-4630

37 Campbell A C, Palmer M R, Klinkhammer G P, et al. Chemistry of hot springs on the Mid-Atlantic Ridge. Nature, 1988, 335: 514-519

38 Allen D E, Seyfried J W E. REE controls in ultramafic hosted MOR hydrothermal systems: An experimental study at elevated temperature and pressure. Geochim Cosmochim Acta, 2005, 69: 675-683

39 Bau M. Rare-earth element mobility during hydrothermal and metamorphic fluid-rock interaction and the significance of the oxidation state of europium. Chem Geol, 1991, 93: 219-230

40 Chiu C L, Song S R, Hsieh Y C, et al. Volcanic characteristics of Kueishantao in northeast Taiwan and their implications. Terr Atmos Ocean Sci, 2010, 21: 575-585

41 Chen C H, Lee T, Shieh Y N, et al. Magmatism at the onset of back-arc basin spreading in the Okinawa Trough. J Volcanol Geotherm Res, 1995, 69: 313-322

42 Chen Y G, Wu W S, Chen C H, et al. A date for volcanic eruption inferred from a siltstone xenolith. Quat Sci Rev, 2001, 20: 869-873

43 Yeh Y H, Lin C H, Roecker S W. A study of upper crustal structures beneath northeastern Taiwan: Possible evidence of the western extension of Okinawa Trough. Proc Geol Soc China, 1989, 32: 139-156

44 Chung S L, Wang S L, Shinjo R, et al. Initiation of arc magmatism in an embryonic continental rifting zone of the southernmost part of Okinawa Trough. Terr Nova, 2000, 12: 225-230

45 Chen C T A, Wang B J, Huang J F, et al. Investigation into extremely acidic hydrothermal fluids off Kueishan Tao, Taiwan, China. Acta Oceanol Sin, 2005, 24: 125-133

46 Yang T F, Lan T F, Lee H F, et al. Gas compositions and helium isotopic ratios of fluid samples around Kueishantao, NE offshore Taiwan and its tectonic implications. Geochem J, 2005, 39: 469-480

47 Chen C T A, Zeng Z G, Kuo F W, et al. Tide-influenced acidic hydrothermal system offshore NE Taiwan. Chem Geol, 2005, 224: 69-81

48 刘长华, 曾志刚, 殷学博, 等. 台湾岛东北部龟山岛附近海域自然硫烟图体的基本特征研究. 台湾海峡, 2006, 25: 309-317

49 曾志刚, 刘长华, 陈镇东, 等. 台湾岛东北部龟山岛热液区自然硫烟图体的成因. 中国科学 D 辑：地球科学, 2007, 37: 1134-1140 
50 刘长华, 曾志刚. 龟山岛附近海底热液自然硫烟图体的硫同位素研究. 海洋与湖沼, 2007, 38: 118-123

51 Zeng Z G, Chen C T A, Yin X B, et al. Origin of native sulfur ball from the Kueishantao hydrothermal field offshore northeast Taiwan-Evidence from trace and rare earth element composition. J Asian Earth Sci, 2011, 40: 661-671

52 郭富雯. 龟山岛海底热液活动初步调查. 硕士学位论文. 高雄: 台湾中山大学, 2001. 12-15

53 刘刚. 海相碳酸盐-稀土元素共沉淀过程中的分异作用研究. 博士学位论文. 青岛: 中国科学院海洋研究所, 2008.134

54 Sun S S, McDonough W F. Chemical and isotopic systematics of oceanic basalts: Implications for mantle composition and processes. Geol Soc Spec Publ, 1989, 42: 313-345

55 Park S H, Lee S M, Kamenov G D, et al. Tracing the origin of subduction components beneath the South East rift in the Manus Basin, Papua New Guinea. Chem Geol, 2010, 269: 339-349

56 曾志刚. 海底热液地质学. 北京: 科学出版社, 2011. 289-300

57 Serrano M J G, Sanz L F A, Nordstrom D K. REE speciation in low-temperature acidic waters and the competitive effects of aluminum. Chem Geol, 2000, 165: 167-180

58 Wood S. The aqueous geochemistry of the rare-earth elements and yttrium 2 . Theoretical predictions of speciation in hydrothermal solutions to $350^{\circ} \mathrm{C}$ at saturation water vapor pressure. Chem Geol, 1990, 88: 99-125 